\title{
Effects of anxiety on the long-term course of depressive disorders ${ }^{\dagger}$
}

William Coryell, Jess G. Fiedorowicz, David Solomon, Andrew C. Leon, John P. Rice and Martin B. Keller

\section{Background}

It is well established that the presence of prominent anxiety within depressive episodes portends poorer outcomes. Important questions remain as to which anxiety features are important to outcome and how sustained their prognostic effects are over time.

\begin{abstract}
Aims
To examine the relative prognostic importance of specific anxiety features and to determine whether their effects persist over decades and apply to both unipolar and bipolar conditions.
\end{abstract}

\section{Method}

Participants with unipolar $(n=476)$ or bipolar $(n=335)$ depressive disorders were intensively followed for a mean of 16.7 years $($ s.d. $=8.5$ ).

\section{Results}

The number and severity of anxiety symptoms, but not the presence of pre-existing anxiety disorders, showed a robust and continuous relationship to the subsequent time spent in depressive episodes in both unipolar and bipolar depressive disorder. The strength of this relationship changed little over five successive 5 -year periods.

\section{Conclusions}

The severity of current anxiety symptoms within depressive episodes correlates strongly with the persistence of subsequent depressive symptoms and this relationship is stable over decades.

\section{Declaration of interest}

A.C.L. has served on Data Safety Monitoring Boards for AstraZeneca, Dainippon Sumitomo Pharma America, Pfizer, Merck, Neuronetics and Vanda, and served as a consultant to the FDA, NIMH, Avera, Cyberonics, Eli Lilly, Schering Plough and Takeda. M.B.K. has received honoraria from and/ or served as a consultant or on advisory boards with: Abbott, CENEREX, Cephalon, Cypress Bioscience, Cyberonics, Forest Laboratories, Medtronic, Organon, Neuronetics, Novartis, Pfizer, Solvay, wyeth, Shire; he has received great support from Pfizer and Wyeth.
Many longitudinal studies of depressive disorders have associated comorbid anxiety with poorer outcomes as reflected in a lower likelihood of treatment response, ${ }^{1-7}$ longer times to recovery from index episodes ${ }^{8-10}$ and greater amounts of depressive morbidity over time. ${ }^{11,12}$ Comorbid anxiety may manifest with the occurrence of panic attacks, with an accompanying diagnosis of an anxiety disorder or with one or more of a variety of anxiety symptoms such as fearfulness, somatic preoccupation, worrying, obsessions or compulsions, phobias and depersonalisation. However, most efforts to characterise the prognostic effects of anxiety on depressive disorders have limited measures to only one or two of these features and have left open the question of which of the many manifestations of anxiety have predictive importance. Previous studies have also not tested whether anxiety symptoms per se portend poorer outcomes or, instead, whether anxiety symptoms simply reflect a general severity of symptoms, inclusive of other depressive features, that is the more prognostically important factor. Finally, because studies have confined their samples to either major depressive disorder or bipolar disorder, the applicability of any set of findings across both disorders has also not been tested.

Our group recently described the effects of four measures of baseline anxiety on long-term depressive and manic/hypomanic morbidity in bipolar disorder - a lifetime history of anxiety disorders, current panic attacks and the severity of 'psychic' and 'somatic' anxiety within the index episode. ${ }^{13}$ Results showed that the polarity of the index episode and a combination of psychic and somatic anxiety severity independently predicted depressive

†See editorial, pp. 179-181, this issue. morbidity such that those who had both a purely manic index episode and anxiety levels below the overall median experienced only one-quarter of the depressive morbidity experienced by those who began follow-up with a depressive index episode and anxiety levels greater than the median. The following analysis was undertaken to test the long-term prognostic significance of a more extensive array of anxiety dimensions as they occur within depressive states, to determine which anxiety features are prognostically important, to test whether the severity of other depressive symptoms have similar prognostic significance, and to compare the applicability of results across bipolar and unipolar groups.

\section{Method}

\section{Participants}

Between 1978 and 1981, inclusive, the National Institute of Mental Health Collaborative Depression Study (CDS) recruited in-patients and out-patients in current episodes of mania, major depression or schizoaffective disorder as they sought treatment at any of five academic centres: Massachusetts General Hospital in Boston; New York State Psychiatric Institute and Columbia Presbyterian Hospital in New York City; Rush Presbyterian Hospital in Chicago; Washington University in St Louis; and the University of Iowa Hospitals and Clinics in Iowa City. All met Research Diagnostic Criteria (RDC) ${ }^{14}$ for one of the requisite diagnoses, and were 18 years or older, English speaking and White. All participants provided informed consent. Recruitment did not influence the treatment that participants received and treatment was naturalistic throughout follow-up. 
In the present analysis participants designated as having bipolar I or bipolar II disorder had current or past episodes of mania, hypomania or schizoaffective mania at the time of the baseline evaluation or, if not, had experienced one or more of those syndromes during follow-up. The RDC definition of the mainly affective subtype of schizoaffective disorder is equivalent to DSM-IV ${ }^{15}$ criteria for bipolar or major depressive disorder with mood-incongruent psychotic features and participants with this diagnosis are therefore included in the present analysis. The analysis was restricted to individuals whose intake episode included a depressive phase preceding the baseline assessment and who completed at least one follow-up interview.

\section{Procedures}

Carefully trained professional raters administered the Schedule for Affective Disorders and Schizophrenia $\left(\right.$ SADS) ${ }^{16}$ as the core diagnostic instrument. In contrast to instruments designed principally for diagnostic screening, the SADS individually characterises a wide scope of symptoms with explicitly anchored, 6 -point severity ratings such that ' 3 ' indicated the presence of a symptom for diagnostic purposes and ' 6 ' the symptom at its most severe. Item ratings reflected an integration of information from participant interview, medical record review and informants when available.

Follow-up assessments took place twice weekly for the first 5 years after intake and annually thereafter. The Longitudinal Interval Follow-up Evaluation (LIFE) ${ }^{17}$ and subsequent, briefer iterations - the LIFE-II and SLICE (details available on request from the author) - used information from participant interview and medical record review to track all RDC disorders active at intake or at any time during follow-up. Interviewers guided participants to identify points in the follow-up interval at which symptoms had changed significantly. Participants then quantified symptom levels present between those change points. Scores for the major affective syndromes of major depression, mania, schizoaffective depression or schizoaffective mania ranged from 1 to 6 , with ' 1 ' indicating no symptoms, ' 2 ' the presence of one or two symptoms to a mild degree, ' 3 ' and ' 4 ' the continued presence of any episode with less than the number of symptoms necessary for an initial diagnosis, ' 5 ' a full syndrome and ' 6 ' a relatively severe full syndrome. Hypomania, minor depression and intermittent depressive disorder symptoms were rated on 3 -point scales in which ' 3 ' indicated a full syndrome. Depressive symptoms were assessed as present for any week with a score of ' 3 ' on the 3-point scales or ' 3 ' or more on the 6-point scales.

The present analyses focused particularly on the eight SADS anxiety symptoms assessed at study intake. The SADS rated individual symptom severity for the week preceding the interview and for the worst week in the current episode. The latter was used in the present analyses.

(a) Worrying: 'brooding, painful preoccupation and inability to get mind off present thoughts'.

(b) Somatic anxiety: extent to which a participant has experienced the physiological concomitants of anxiety that are typical of panic attacks but that have occurred outside of panic attacks (i.e. headaches, stomach cramps, palpitations, shortness of breath, diarrhoea or muscle tension).

(c) Psychic anxiety: 'subjective feelings of anxiety, fearfulness or apprehension, excluding panic attacks whether focused on specific concerns or not'.

(d) Phobia: fears of objects, situations or activities - 'like crowds, certain animals, heights, being alone, being closed in, going out alone or certain ways of travelling. (e) Somatic preoccupation: the participant's degree of preoccupation with real or imagined physical illness or disability.

(f) Depersonalisation: the frequency with which participants reported having felt 'as if you were outside of your own body, or as if a part does not belong to you, or that you are physically cut-off from people, or floating, or like you are in a dream'.

(g) Obsessions and compulsions: a single score that encompassed the intensity and frequency of obsessions and compulsions and the resulting impairment.

(h) Panic attacks: rated as absent, 'questionable' or present in the current episode.

Our analyses tested anxiety symptom ratings individually and, again, in aggregate as the sum of all anxiety symptom scores. To explore the importance of anxiety symptom diversity the analyses also tested a symptom count, the sum of anxiety symptoms rated as present or absent according to the SADS item cut-off of ' 3 ' or more.

The prognostic importance of individual RDC anxiety disorders - panic, obsessive-compulsive, generalised anxiety and phobic - was tested separately. Diagnostic hierarchies that gave precedence to major affective syndromes over anxiety disorders were omitted from the DSM-III-R ${ }^{18}$ and DSM-IV but were present in the earlier diagnostic systems of Feighner et al, ${ }^{19}$ the RDC $^{14}$ and the DSM-III. ${ }^{20}$ Thus, the RDC assigned a diagnosis of panic disorder or obsessive-compulsive disorder only if the panic attacks, or the obsessions and compulsions, had occurred either at least 2 months before an episode of major depression, mania or schizoaffective disorder had begun, or at least 2 months after it had ended. Consequently, the RDC, when applied to groups with mood disorders, produces lower rates of anxiety disorder diagnoses than does DSM-IV. In further contrast to DSM-IV, the RDC did not include the categories of posttraumatic stress disorder or acute stress disorder.

To determine whether the severity of anxiety symptoms had predictive significance beyond the degree to which they simply correlated with overall depressive symptom severity, we chose to compare the prognostic importance of a symptom cluster that has been thought to have prognostic importance but that has minimal overlap with anxiety symptoms, that of the endogenous subtype. For use here this measure was restricted to symptoms rated for severity on 6-point scales and did not include morning dysphoria, lack of reactivity and distinct quality of mood. The resulting symptom measure was thus the sum of ratings for selfreproach, guilt, anhedonia, psychomotor agitation, psychomotor retardation, anorexia and weight loss. Because this grouping omitted three of the original ten symptoms that comprised the endogenous subtype, the following will refer to these as 'core depressive symptoms'.

\section{Data analysis}

Generalised linear models (GLM) (SPSS version 18 for Mac) were used to examine relationships between baseline demographic, diagnostic and symptom severity measures, and the subsequent proportion of observed follow-up weeks during which participants had clinically significant symptoms of major depressive disorder, schizoaffective depression, minor depressive disorder or intermittent depressive disorder. Because the distribution of this proportion ( $p$ ) is right-skewed we chose to use a logit transformation, $\log (\mathrm{p} / 1-\mathrm{p})$, as this best approximated a normal distribution for our dependent variable. In addition to confidence intervals, the GLM generates a $\beta$-value that quantifies the degree to which changes in the independent variable 
correspond to changes in the transformed dependent variable. To facilitate comparisons of $\beta$-values derived from combined symptom scores of variable ranges we condensed each to the 6-point range that characterised each of the individual anxiety symptoms. Baseline age and gender were tested alone to be included in subsequent models if they were predictive of depressive morbidity. Non-parametric tests were used for follow-up group comparisons and for correlations. A two-tailed $\alpha=0.05$ was used throughout.

\section{Results}

\section{Unipolar major depressive disorder}

Of the 476 participants with unipolar depressive disorder (the unipolar group) with at least 6 months of follow-up, 126 (26.5\%) were known to have died subsequently. Of the remaining $350,215(61.4 \%)$ completed 20 years of follow-up and 145 $(41.4 \%)$ completed 25 years. Those followed to 25 years were more likely to have a baseline diagnosis of phobic disorder (18 $(12.4 \%))$ than those with shorter follow-ups (9 (4.4\%)) $\left(\chi^{2}=7.7, P=0.006\right)$ and, accordingly, the two groups differed by severity of phobic anxiety during the index episode; mean values were 2.4 (s.d. $=1.5)$ and 2.1 (s.d. $=1.3)$, respectively $(t=2.4$, d.f. $=310, P=0.019)$. Those who completed 25 years of followup did not otherwise differ significantly from non-completers for the variables listed in Table 1 with the exception of years of follow-up.

Older age at intake ( $\beta=0.021,95 \%$ CI $0.002-0.039, P=0.039$ ), but not gender, was associated with a greater proportion of follow-up time with depressive symptoms and was therefore included in GLM analyses for the unipolar group. Only one in six participants met RDC for any lifetime anxiety disorder although nearly one-third described panic attacks during the index depressive episode (Table 1). Lifetime diagnoses of anxiety disorders were not significantly associated with subsequent time in depressive episodes whether the diagnoses were considered individually (panic disorder, obsessive-compulsive disorder, phobic disorder or generalised anxiety disorder), or together as the presence or absence of any anxiety disorder $(\beta=0.227,95 \%$ CI -0.960 to $0.371, P=0.542)$. Likewise, the presence or absence of other disorders (alcoholism, antisocial personality disorder or drug dependence) was not significantly predictive of time with depressive symptoms whether entered individually or together as any non-mood, non-anxiety disorder $(\beta=0.548,95 \% \mathrm{CI}-1.15$ to $0.050, P=0.072$ ).

Of the eight baseline anxiety measures individually tested, all were positively related to the proportion of follow-up weeks in depressive episodes and six were significantly so (Table 2 ). With
Table 1 Demographic and clinical measures in

participants with bipolar (I and II) and major depressive

disorder with an epidsode of depression at intake

Major depressive Bipolar disorder disorde $(n=476) \quad(n=335)$

\begin{tabular}{|lcc|}
\hline Age, mean (s.d.) & & \\
At intake & $39.5(14.8)$ & $36.3(13.2)$ \\
At onset & $27.3(13.4)$ & $22.5(9.3)$ \\
\hline Years of follow-up, mean (s.d.) & $15.8(8.6)$ & $18.0(8.1)$ \\
\hline Anxiety measures, mean (s.d.) & & \\
Worrying & $4.2(1.3)$ & $4.0(1.4)$ \\
Phobic concerns & $2.1(1.3)$ & $2.2(1.4)$ \\
Obsessions/compulsions & $1.5(0.9)$ & $1.6(1.1)$ \\
Psychic anxiety & $3.8(1.4)$ & $3.8(1.5)$ \\
Somatic anxiety & $3.2(1.6)$ & $3.0(1.5)$ \\
Depersonalisation & $1.7(1.3)$ & $1.9(1.5)$ \\
Somatic preoccupation & $2.1(1.5)$ & $2.0(1.4)$ \\
\hline Gender, female: $n$ (\%) & $283(59.6)$ & $204(60.7)$ \\
\hline Panic attacks, $n$ (\%) & $155(32.6)$ & $99(29.5)$ \\
\hline Lifetime diagnosis, anxiety disorder: $n(\%)$ & & \\
Panic & $24(5.1)$ & $16(4.8)$ \\
Generalised anxiety disorder & $33(6.9)$ & $19(5.7)$ \\
Obsessive-compulsive disorder & $9(1.9)$ & $9(2.7)$ \\
Phobic disorder & $36(7.6)$ & $20(6.0)$ \\
Any anxiety disorder & $80(16.8)$ & $50(14.9)$ \\
\hline Lifetime diagnoses, other: $n$ (\%) & $11(2.3)$ & $6(1.8)$ \\
Antisocial personality & $128(26.9)$ & $90(26.8)$ \\
Alcoholism & $30(6.3)$ & $33(9.8)$ \\
Drug dependence & & \\
& & \\
& & \\
& & \\
& & \\
\hline
\end{tabular}

these six symptoms in the model together with age, only the severity of obsessive-compulsive symptoms was significantly predictive of depressive morbidity $(\beta=0.404,95 \%$ CI 0.101 to $0.706, P=0.009)$ although a borderline relationship remained for depersonalisation $(\beta=0.201,95 \%$ CI -0.009 to 0.411 , $P=0.061)$. Of 477 participants, $54(11.3 \%)$ had rating of 3 or more, indicative of the presence of the symptom. When the sum of all anxiety symptoms other than obsessions and compulsions were entered into the model both the sum of the other symptoms ( $\beta=0.336,95 \%$ CI $0.122-0.550, P=0.002)$ and the obsessivecompulsive symptom rating $(\beta=0.404,95 \%$ CI $0.102-0.705$, $P=0.009)$ were predictive.

Individual anxiety symptom scores were broadly intercorrelated such that all eight symptoms significantly covaried with at least five of the other symptoms. None of the 28 intercorrelations exceeded Spearman rho values of 0.37 , however. The two symptoms that had the highest mean correlations with other

\begin{tabular}{|c|c|c|c|c|c|c|}
\hline & \multicolumn{3}{|c|}{ Major depressive disorder ${ }^{a}(n=476)$} & \multicolumn{3}{|c|}{ Bipolar disorder $(n=335)$} \\
\hline & $\beta$ & $95 \% \mathrm{Cl}$ & $P$ & $\beta$ & $95 \% \mathrm{Cl}$ & $P$ \\
\hline Worrying & 0.129 & -0.088 to 0.346 & 0.245 & -0.016 & -0.206 to 0.175 & 0.873 \\
\hline Phobias & 0.133 & -0.075 to 0.340 & 0.212 & 0.280 & 0.088 to 0.473 & 0.004 \\
\hline Obsessions/compulsions & 0.507 & 0.209 to 0.805 & 0.001 & 0.393 & -0.145 to 0.640 & 0.002 \\
\hline Somatic anxiety & 0.214 & 0.044 to 0.384 & 0.014 & 0.288 & 0.112 to 0.464 & 0.001 \\
\hline Psychic anxiety & 0.260 & 0.071 to 0.449 & 0.007 & 0.236 & 0.063 to 0.410 & 0.008 \\
\hline Somatic preoccupation & 0.196 & 0.012 to 0.380 & 0.037 & 0.290 & 0.097 to 0.484 & 0.003 \\
\hline Depersonalisation & 0.268 & 0.058 to 0.479 & 0.012 & 0.188 & 0.009 to 0.368 & 0.040 \\
\hline Panic attacks & 0.458 & 0.153 to 0.762 & 0.003 & 0.242 & -0.063 to 0.547 & 0.120 \\
\hline
\end{tabular}


symptoms were panic attacks (mean $r=0.23$ (s.d.=0.058)) and somatic anxiety (mean $r=0.19$ (s.d. $=0.113)$ ); the two with the lowest mean correlations with other symptoms were the obsessions-compulsions item (mean $r=0.12$ (s.d.=0.054)) and depersonalisation (mean $r=0.09$ (s.d. $=0.064)$ ).

Because the severity of all anxiety symptoms appeared prognostically important, and because they were moderately although consistently intercorrelated, we summed the eight symptoms for further analysis. The resulting total anxiety symptom severity score was related in a linear fashion to subsequent time with depressive symptoms $(\beta=0.479,95 \% \mathrm{CI}$ $0.239-0.719, \quad P<0.0001)$. Mean (s.d.) proportions of weeks depressed in the lowest to highest anxiety symptom quartiles were 0.31 (s.d. $=0.32), 0.39$ (s.d. $=0.31), 0.49$ (s.d. $=0.34$ ) and 0.51 (s.d. $=0.35)(F=9.7$, d.f. $=3,472, P<0.0001)$.

When similarly tested, the core depressive symptom score was also associated with time in depressive episodes ( $\beta=0.347,95 \%$ CI $0.086-0.608, P=0.009$ ). However, in a model together with the anxiety symptom severity score the former was substantially more predictive $(\beta=0.424,95 \%$ CI $0.176-0.672, P<0.0001)$ than the latter $(\beta=0.231,95 \%$ CI $0.036-0.498, P=0.090)$.

\section{Bipolar major depression}

Of 335 participants with bipolar depressive disorder (the bipolar group) with at least 6 months of follow-up, 70 (20.9\%) were known to have died subsequently. Of the remaining 265, 181 $(68.3 \%)$ completed 20 years of follow-up and 144 (54.3\%) completed 25 years. Those who completed 25 years of follow-up were more likely to be female $(103(71.5 \%))$ than were those who did not $(67(55.4 \%))\left(\chi^{2}=7.5\right.$, d.f. $\left.=1, P=0.006\right)$. With the exception of years of follow-up, completers otherwise did not differ significantly from non-completers for the variables listed in Table 1.

As noted, this analysis included only participants with bipolar disorder with an index episode that had included a depressive phase by the time of intake. Those with bipolar I did not differ from those with bipolar II in the proportion of time with depressive symptoms during follow-up (mean values were 0.34 (s.d. $=0.29)$ and 0.37 (s.d. $=0.28)$, respectively) and these groups were therefore combined in subsequent analyses. Neither age nor gender was associated with the subsequent proportion of time in depressive episodes within the bipolar group and these variables were thus not included in the GLM models of anxiety symptoms.

Six of the eight anxiety measures were predictive of subsequent time in depressive episodes in univariate analyses (Table 2) and, when tested together in the same model, only obsessive-compulsive severity was significantly related to subsequent time depressed $(\beta=0.273,95 \%$ CI $0.022-0.524$, $P=0.031)$. An obsession or compulsion was considered present in 46 individuals (13.7\%). When tested together, both the obsessive-compulsive severity score $(\beta=0.290$, 95\% CI 0.038 $0.542, P=0.024)$ and the anxiety score minus the obsessivecompulsive rating ( $\beta=0.325,95 \%$ CI $0.126-0.525, P=0.001$ ) were important.

Of the four RDC anxiety disorders only the presence of lifetime obsessive-compulsive disorder was associated with time in depressive episodes in a GLM analysis $(\beta=2.375,95 \%$ CI -0.402 to $-0.725, P=0.005)$. Only nine $(2.7 \%)$ of the bipolar group had this diagnosis but these nine were in depressive episodes for a mean proportion of 0.66 (s.d. $=0.30)$ weeks in comparison with a mean of 0.34 (s.d. $=0.28$ ) weeks for those without this diagnosis $(t=2.8$, d.f. $=333, P=0.005)$.

The total anxiety symptom score was again robustly predictive $(\beta=0.563,95 \%$ CI $0.342-0.784, \quad P<0.0001)$ and the mean proportions of follow-up weeks depressed across the quartiles of anxiety severity were 0.34 (s.d. $=0.31), 0.37$ (s.d. $=0.29), 0.41$ (s.d.=0.31), and $0.52 \quad$ (s.d. $=0.31) \quad(F=5.3, \quad$ d.f. $=3,329$, $P=0.001)$. The core depressive symptom score was also predictive when tested alone $(\beta=0.318,95 \%$ CI $0.076-0.560, P=0.010)$. When tested together, though, the anxiety symptom severity score remained predictive ( $\beta=0.535,95 \%$ CI 0.290 to $0.781, P<0.0001$ ) but the depressive symptom score did not $(\beta=0.068,95 \%$ $\mathrm{CI}=-0.194$ to $0.330, P=0.610$ ).

Also, as in the major depressive disorder group, individual symptom scores were widely intercorrelated and only 1 of the 28 correlations was insignificant. None of the Spearman rho values however, exceeded 0.36 . The two symptoms with the highest mean correlation with other symptoms were somatic anxiety (mean $r=0.239$ (s.d. $=0.047$ ) ) and phobias (mean $r=0.224$ (s.d. $=$ $0.063)$ ); the two least highly correlated with all other symptoms were the obsessive-compulsive rating (mean $r=0.153$ (s.d.= $0.033)$ ) and depersonalisation (mean $r=0.159$ (s.d. $=0.063)$ ).

\section{Unipolar and bipolar groups combined}

Because the effects of baseline anxiety levels on subsequent depressive morbidity were similar in the unipolar and bipolar groups they were combined to further determine the correlational structures of the anxiety symptom array and the quantitative relationship of symptom severity to depressive morbidity as follow-up progressed. With only one exception (worry $v$. depersonalisation) each of the eight anxiety symptoms correlated significantly with each of the others with all $\alpha$ values $<0.01$, a mean rho value of 0.178 (s.d. $=0.078$ ) and a range of $0.04-0.36$.

The proportion of time in depressive episodes correlated with the total symptom severity score $(r=0.24, P<0.0001)$ and the count of anxiety symptoms $(r=0.22, P<0.0001)$ to similar degrees. With both measures in a GLM model, however, the severity measure $(\beta=0.111,95 \%$ CI 0.027 to $0.195, P=0.009)$ but not the symptom number $(\beta=<0.032,95 \%$ CI -0.315 to $0.251, P=0.825)$ emerged as significant.

To determine the persistence of relationships between baseline anxiety severity and later depressive morbidity, baseline anxiety severity quartiles were plotted against the proportion of observed weeks in depressive episodes for each of five 5-year periods for those participants who completed each of these periods (Fig. 1). The proportion of weeks in depressive episodes increased progressively and significantly across baseline anxiety severity quartiles in each of the 5-year periods and differences between the lowest and highest baseline quartiles changed little over the 25-year span. In contrast, baseline core depressive symptom quartiles corresponded to significantly increasing time with depressive symptoms in the first 5-year period $(F=5.8$, d.f. $=3, P=0.001)$ but the effect faded in the second 5 -year period $(F=2.6$, d.f. $=3, P=0.054)$ and was not significant in any of the subsequent three 5 -year periods.

\section{Discussion}

\section{Strengths and weaknesses}

Strengths of this study lie in its size, the length and intensity of the prospective assessments, and in the thoroughness with which baseline clinical phenomena were assessed. These qualities afforded an unmatched potential to measure the impact of anxiety symptoms, individually and in aggregate, on depressive morbidity over a substantial portion of the adult lifespan. The CDS shares with other naturalistic studies the absence of control over treatment. Results may have differed if specific treatment algorithms had been followed, but such control is not compatible with long-term observation as study attrition inexorably results in small and atypical samples. One limitation that deserves particular 


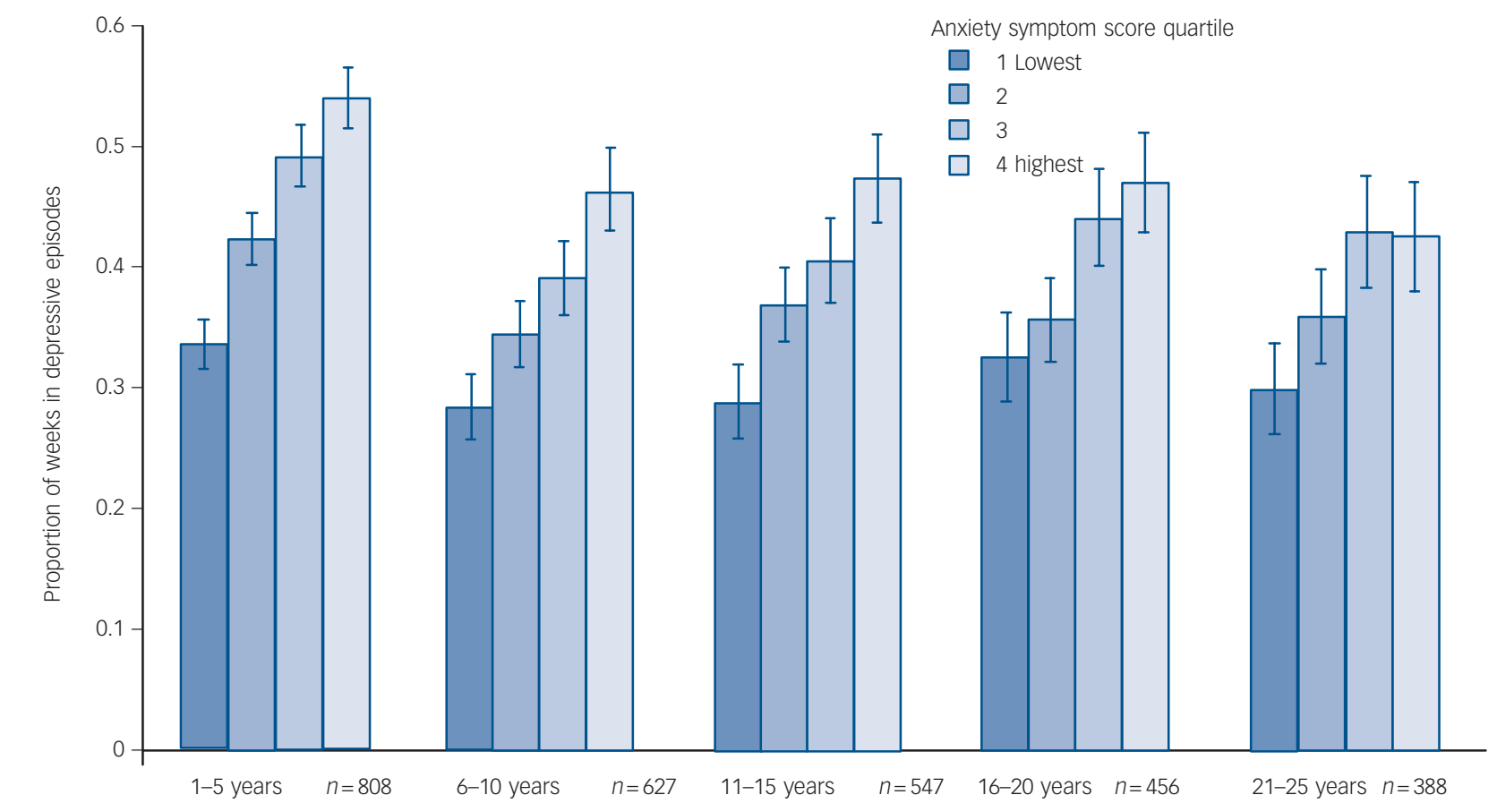

Fig. 1 Baseline anxiety symptom severity levels and mean (s.e.) proportions of weeks in depressive episodes by follow-up period.

emphasis, though, concerns the settings in which the participants were recruited. Individuals with relatively complex and refractory disorders are overrepresented in tertiary care centres and the CDS made no effort to exclude those with these characteristics. Results therefore may not generalise well to patients seen in primary care settings. This caveat is probably more relevant to the degree of depressive morbidity seen in the entire sample than to the relationships between that morbidity and baseline anxiety measures.

\section{Main findings}

With the exception of worrying, each of the eight anxiety symptoms was significantly related to subsequent, long-term depressive morbidity in either unipolar or bipolar major depression and five were significantly so in both groups. Obsessive-compulsive symptom severity was consistently the most predictive but this symptom was relatively infrequent and the other anxiety symptoms taken together were robustly predictive when tested with obsessive-compulsive severity in the model. Notably, the obsessive-compulsive score was only weakly correlated with other anxiety symptoms and the presence of a lifetime diagnosis of obsessive-compulsive disorder was the only anxiety disorder to be associated with significantly increased depressive morbidity during follow-up, albeit only in the bipolar group. Thus, although a global severity rating of anxiety symptoms generally has considerable prognostic importance, the presence of obsessions or compulsions in a depressive episode appears to deserve special attention.

Within the bipolar group the nine participants with preexisting obsessive-compulsive disorder experienced significantly greater depressive morbidity during follow-up. Otherwise, anxiety disorder comorbidity, here defined as the lifetime coexistence of a major mood disorder with an anxiety syndrome that manifests outside of mood episodes, was not associated with subsequent time in depressive episodes. In contrast, anxiety symptoms that occurred within the index depressive episodes were highly predictive. This speaks to the validity of the older hierarchical diagnostic symptoms that recognised a difference between anxiety symptoms that are confined to mood episodes and those that appear at times when mood symptoms are inactive. Indeed, older studies that used such hierarchies found that the relatives of probands with panic disorder and secondary depression (coming after panic disorder is established) were not at increased risk for major depressive disorder when compared with the relatives of controls who were well. ${ }^{10,21-24}$ Nor were the relatives of probands with major depressive disorder whose panic attacks were confined to depressive episodes more likely to have panic disorder than were relatives with no history of panic attacks. ${ }^{10,25}$ Follow-up studies have further supported a diagnostic distinction based on temporal relationships between anxiety and depressive syndromes. Participants who experienced panic attacks only during depressive episodes rarely went on to experience freestanding panic disorder $^{10,26}$ and were much more likely to be without anxiety symptoms on follow-up than were participants whose panic attacks had appeared before any depressive syndrome had developed. $^{27}$

Severity ratings for the eight anxiety symptoms were widely but modestly intercorrelated in both unipolar and bipolar groups and this probably accounts for the failure of most individual symptoms to emerge as significant predictors when entered together in GLM analyses. Moreover, the number of anxiety symptoms, each scored dichotomously, predicted depressive morbidity nearly as well as the sum of individual symptom severity ratings. Together these observations argue for a global rating of anxiety present during depressive episodes that gives weight to both the severity and the variety of anxiety phenomena present.

\section{Implications}

The finding that the severity of core depressive symptoms had relatively modest and transient relationships with subsequent depressive morbidity indicates that the anxiety symptoms have a particular prognostic importance in depressive disorders beyond their association with overall symptom severity. The constancy 
of this relationship over a 25 -year follow-up period is evidence that prominent and diverse anxiety symptoms characterise not simply a given episode but, instead, an individual's lifetime depressive disorder. It may thus be useful in characterising phenotypes for genetic research although the lack of a clear threshold with which to identify highly anxious groups is problematic.

A well-established relationship exists between the severity of depressive symptoms, lower placebo response rates and greater drug-placebo differences in unipolar major depressive disorder. ${ }^{28}$ That a similar relationship has been demonstrated between anxiety and the likelihood of drug-placebo difference in bipolar depression ${ }^{7}$ underscores the importance of further analyses of such data to establish whether measures of anxiety might be more useful than overall depressive symptom severity in selection of individuals for pivotal antidepressant trials and in clinical decisions to initiate antidepressant therapy.

\footnotetext{
William Coryell, MD, Department of Psychiatry, Roy J. and Lucille A. Carver College
of Medicine, University of lowa Hospitals and Clinics, lowa and Department of of Medicine, University of lowa Hospitals and Clinics, lowa and Department of
Psychiatry, Columbia University College of Physicians and Surgeons, New York; Psychiatry, Columbia University College of Physicians and Surgeons, New York;
Jess G. Fiedorowicz, MD, MS, Department of Psychiatry, Roy J. and Lucille A. Carve College of Medicine, University of lowa Hospitals and Clinics, Iowa; David Solomon MD, Department of Psychiatry and Human Behavior, The Warren Alpert Medical School of Brown University, Providence, Rhode Island; Andrew C. Leon, PhD, Department of Psychiatry, Columbia University College of Physicians and Surgeons, New York; John P. Rice, PhD, Department of Psychiatry, Washington University School of Medicine, St Louis, Missouri; Martin B. Keller, MD, Department of Psychiatry and Human Behavior, The Warren Alpert Medical School of Brown University, Providence, Rhode Island, USA

Correspondence: William Coryell, MD, Roy J. and Lucille A. Carver College of Medicine, University of Iowa Hospitals and Clinics, 200 Hawkins, Iowa City, IA 52242, USA. Email: william-coryell@uiowa.edu

First received 3 Mar 2011, final revision 10 May 2011, accepted 16 Jun 2011
}

\section{Acknowledgements}

Conducted with the current participation of the following investigators: M. B. Keller, MD (Chairperson, Providence, RI); W. Coryell MD (Co-Chairperson, Iowa City, IA); D. A. Solomon, MD (Providence, RI); W. Scheftner, MD (Chicago, IL); J. Endicott, PhD, A. C. Leon, PhD, J. Loth, MSW (New York, NY) and J. Rice, PhD (St. Louis, MO). Other current contributors include H.S. Akiskal, MD, J. Fawcett, MD, L. L. Judd, MD, P. W. Lavori, PhD, J. D. Maser, PhD and T. I. Mueller, MD. This manuscript has been reviewed by the Publication Committee of the Collaborative Depression Study and has its endorsement. The data for this manuscript came from the National Institute of Mental Health (NIMH) Collaborative Program on the Psychobiology of Depression - Clinical Studies. The Collaborative Program was initiated in 1975 to investigate nosologic, genetic, family, prognostic, and psychosocial issues of mood disorders, and is an ongoing long-term multidisciplinary investigation of the course of mood and related affective disorders. The original principal and co-principal investigators were from five academic centres and included Gerald Klerman, MD (deceased, Co-Chairperson), Martin Keller, MD, Robert Shapiro, MD (deceased) (Massachusetts General Hospital, Harvard Medical School), Eli Robbins, MD (deceased), Paula Clayton, MD, Theodore Reich, MD (deceased), Amos Wellner, MD (deceased) (Washington University Medical School), Jean Endicott, PhD, Robert Spitzer, MD, (Columbia University), Nancy Andreasen, MD, PhD, William Coryell, MD, George Winokur, MD (deceased) (University of lowa), Jan Fawcett, MD, William Scheftner, MD, (RushPresbyterian-St. Luke's Medical Center). The NIMH Clinical Research Branch was an active Presbyterian-St. Luke's Medical Center). The NIMH Clinical Research Branch was an active
collaborator in the origin and development of the Collaborative Program with Martin M. Katz, PhD, Branch Chief as the Co-Chairperson and Robert Hirschfeld, MD as the Program Coordinator. Other past collaborators include J. Croughan, MD, M. T. Shea, PhD, R. Gibbons, PhD, M. A. Young, PhD, D. C. Clark, PhD.

\section{Funding}

Data for this manuscript were generated by the Collaborative Program on the Psychobiology of Depression - Clinical Studies, funded by the National Institute of Mental Health.

\section{References}

1 Henry C, Van den Bulke D, Bellivier F, Etain B, Rouillon F, Leboyer M. Anxiety disorders in 318 bipolar patients: prevalence and impact on illness severity and response to mood stabilizer. J Clin Psychiatry 2003; 64: 331-5.

2 Fava M, Rush AJ, Alpert JE, Balasubramani GK, Wisniewski SR, Carmin CN, et al. Difference in treatment outcome in outpatients with anxious versus nonanxious depression: a STAR ${ }^{\star} \mathrm{D}$ report. Am J Psychiatry 2008; 165 : 342-51.

3 Fava M, Uebelacker LA, Alpert JE, Nierenberg AA, Pava JA, Rosenbaum JF. Major depressive subtypes and treatment response. Biol Psychiatry 1997; 42: $568-76$.

4 Feske U, Frank E, Kupfer DJ, Shear MK, Weaver E. Anxiety as a predictor of response to interpersonal psychotherapy for recurrent major depression: an exploratory investigation. Depress Anxiety 1998; 8: 135-41.

5 Feske U, Frank E, Mallinger AG, Houck PR, Fagiolini A, Shear MK, et al. Anxiety as a correlate of response to the acute treatment of bipolar I disorder. Am J Psychiatry 2000; 157: 956-62.

6 Papakostas GI, McGrath P, Stewart J, Charles D, Chen Y, Mischoulon D, et al. Psychic and somatic anxiety symptoms as predictors of response to fluoxetine in major depressive disorder. Psychiatry Res 2008; 30: 116-20.

7 Tohen M, Calabrese J, Vieta E, Bowden C, Gonzalez-Pinto A, Lin D, et al. Effect of comorbid anxiety on treatment response in bipolar depression. J Affect Disord 2007; 104: 137-46.

8 Gaynes BN, Magruder KM, Burns BJ, Wagner HR, Yarnall KS, Broadhead WE. Does a coexisting anxiety disorder predict persistence of depressive illness in primary care patients with major depression? Gen Hosp Psychiatry 1999; 21: 158-67.

9 Clayton PJ, Grove WM, Coryell W, Keller M, Hirschfeld R, Fawcett J. Follow-up and family study of anxious depression. Am J Psychiatry 1991; 148: 1512-7.

10 Coryell W, Endicott J, Andreasen NC, Keller MB, Clayton PJ, Hirschfeld RM, et al. Depression and panic attacks: the significance of overlap as reflected in follow-up and family study data. Am J Psychiatr 1988; 145: 293-300.

11 Coryell W, Endicott J, Winokur G. Anxiety syndromes as epiphenomena of primary major depression: outcome and familial psychopathology. Am J Psychiatry 1992; 149: 100-7.

12 Otto MW, Simon NM, Wisniewski SR, Miklowitz DJ, Kogan JN, Reilly-Harrington NA, et al. Prospective 12-month course of bipolar disorder in out-patients with and without comorbid anxiety disorders. Br J Psychiatry 2006; 189: 20-5.

13 Coryell W, Solomon DA, Fiedorowicz JG, Endicott J, Schettler PJ, Judd LL. Anxiety and outcome in bipolar disorder. Am J Psychiatry 2009; 166: 1238-43.

14 Spitzer RL, Endicott J, Robins E. Research Diagnostic Criteria (3rd edn). Biometrics Research, New York State Department of Mental Hygiene, 1978.

15 American Psychiatric Association. Diagnostic and Statistical Manual of Mental Disorder (4th edn) (DSM-IV). APA, 1994.

16 Endicott J, Spitzer RL. A diagnostic interview: the schedule for affective disorders and schizophrenia. Arch Gen Psychiatry 1978; 35: 837-44.

17 Keller MB, Lavori PW, Friedman B, Nielsen E, Endicott J, McDonald-Scott P et al. The Longitudinal Interval Follow-up Evaluation. A comprehensive method for assessing outcome in prospective longitudinal studies. Arch Gen Psychiatry 1987; 44: 540-8.

18 American Psychiatric Association. Diagnostic and Statistical Manual of Mental Disorders (3rd edn, revised) (DSM-III-R). APA, 1987.

19 Feighner JP, Robins E, Guze SB, Woodruff Jr RA, Winokur G, Munoz R. Diagnostic criteria for use in psychiatric research. Arch Gen Psychiatry 1972; 26: $57-63$.

20 American Psychiatric Association. Diagnostic and Statistical Manual of Mental Disorder (3rd edn) (DSM-III). APA, 1980.

21 Crowe RR, Noyes R, Pauls DL, Slymen D. A family study of panic disorder. Arch Gen Psychiatry 1983; 40: 1065-9.

22 Dealy RS, Ishiki DM, Avery DH, Wilson LG, Dunner DL. Secondary depression in anxiety disorders. Compr Psychiatry 1981; 22: 612-8.

23 Noyes Jr R, Crowe RR, Harris EL, Hamra BJ, Mcchesney CM, Chaudhry DR Relationship between panic disorder and agoraphobia. A family study. Arch Gen Psychiatry 1986; 43: 227-32.

24 Van Valkenburg C, Winokur G, Behar D, Lowry M. Depressed women with panic attacks. J Clin Psychiatry 1984; 45: 367-9.

25 Dindo L, Coryell W. Comorbid major depression and panic disorder: significance of temporal sequencing to familial transmission. J Affect Disord 2004; 82: 119-23.

26 Kessler RC, Stang PE, Wittchen HU, Ustun TB, Roy-Burne PP, Walters EE. Lifetime panic-depression comorbidity in the National Comorbidity Survey. Arch Gen Psychiatry 1998; 55: 801-8.

27 Nutzinger DO, Zapotoczky HG. The influence of depression on the outcome of cardiac phobia (panic disorder). Psychopathology 1985; 18: 155-62.

28 Khan A, Leventhal RM, Khan SR, Brown WA. Severity of depression and response to antidepressants and placebo: an analysis of the Food and Drug Administration database. J Clin Psychopharmacol 2002; 22: 40-5. 